\title{
Consequences of Partial Axotomy for Production of Neurotransmitter Vesicles and Routing of Rapidly Transported Membrane Glycoproteins in the Axonal Tree
}

\author{
Daniel J. Goldberg and Richard T. Ambron \\ Departments of Pharmacology and Anatomy and Cell Biology and Center for Molecular Neurobiology and Behavior, \\ Columbia University, College of Physicians and Surgeons, New York, New York 10032
}

It was found previously that transection of one branch of the bifurcate axon of a giant serotonergic neuron of Aplysia causes a proportionate decrease in the export of transmitter storage vesicles from the cell body, despite the ability of the remaining branch to transport all of the vesicles normally exported (Aletta and Goldberg, 1982). We report here evidence that this downregulation is achieved by a decrease in the production of vesicles, rather than a slowing of the processing of vesicles for export from the cell body. Counts of serotonergic vesicles in the cell body show that the size of the somal pool of vesicles is not increased at a time when their rate of export has been halved. Also, transection of one or both branches of the axon decreases the amount of ${ }^{3} \mathrm{H}$-fucose incorporated during a short period into a putative vesicle membrane glycoprotein but not into other glycoproteins. The results indicate that axotomy should be a useful technique for studying the mechanism of regulation of the synthesis of a secretory organelle.

It was also previously shown that transection of one branch of the axon far from the cell body does not impair the ability of the injured branch to transport serotonergic vesicles, yet the majority of vesicles that would normally enter that branch are nevertheless rerouted into the uninjured branch. We have studied quantitatively the partitioning between the 2 branches, after one has been transected distally, of 6 fucosyl glycoproteins, including the putative vesicle glycoprotein and a glycoprotein whose transport moderately increases after transection. None is selectively routed into one branch or the other, though there is stochastic variation in the composition of material received by axon endings.

The axon and terminals of a neuron, being unable to synthesize macromolecules, depend for their functional and anatomical integrity on a continual supply of organelles and cytoskeletal components from the cell body. During the life of the neuron the activities of these regions change. A developing or regenerating axon is elongating and its endings are involved in pathfinding and target recognition, while mature synaptic terminals are engaged principally in synaptic transmission. It is not clear to what extent these changes in activity are supported by changes in the delivery of materials from the cell body, as opposed to

Received Aug. 19, 1985; revised Nov. 19, 1985; accepted Nov. 27, 1985.

This work was supported by NIH research grants NS 14711 (D.J.G.) and NS 14555 (R.T.A.), NIH Research Career Development Award NS 00350 (R.T.A.), and grants from the Stifel Paralysis Research Foundation and the William J. Matheson Foundation (D.J.G.). We wish to thank Dr. John M. Aletta for participating in some of the early experiments and for helpful comments on the manuscript and Ms. Arline Albala for doing much of the electron microscopy.

Correspondence should be addressed to Dr. Goldberg, Department of Pharmacology, Columbia University, 630 West 168th Street, New York, NY 10032. Copyright (C) 1986 Society for Neuroscience $0270-6474 / 86 / 061712-07 \$ 02.00 / 0$ changes in the use of those materials, but the fact that axotomy typically causes a reduction in the transport of at least some materials associated with synaptic transmission (Aletta and Goldberg, 1982; Boyle and Gillespie, 1970; Hebb and Silver, 1966) and an increase in others associated with growth (Giulian et al., 1980; Skene and Willard, 1981) suggests that changes in delivery may be important.

In examining the regulation of the export of materials from the cell body, we have focused on the transmitter storage vesicle. It was shown previously that the export of serotonergic vesicles in the giant cerebral neuron (GCN) of the marine mollusc $A p l y$ sia californica is regulated, bcing closely coupled to the extent of the peripheral field that must be supplied (Aletta and Goldberg, 1982). One purpose of the work reported here was to examine how the cell body effects this regulation. When the extent of the peripheral field is reduced by axotomy, does the rate of export of transmitter vesicles decrease because their rate of synthesis decreases, or rather because newly synthesized vesicles take longer to exit the cell body? We present here evidence that implicates synthesis as the regulated step.

Regional specialization in the axonal tree must likewise result from differences in the composition of the material delivered to the various branches or from differences in the use of these materials by the branches. It is thus of considerable importance to identify the factors that determine how transported material partitions among the branches of an axon and to determine whether the composition of transported material can differ among branches. The partitioning of serotonergic storage vesicles at the bifurcation of the axon of GCN is influenced by the state of the branch endings (Aletta and Goldberg, 1984). Vesicles tend to move into a branch that has its full complement of synaptic terminals in preference to a branch that has lost some or most of its terminals. We have sought to determine, in the experiments reported here, if other transported materials also behave in this way or whether vesicles are shunted away from the pruned branch selectively. Examining the partitioning of several fucosyl glycoproteins, at least one of which is unlikely to be a constituent of the serotonergic vesicle, we find no evidence for selectivity. Some of these results were reported in preliminary form (Goldberg and Ambron, 1985).

\section{Materials and Methods}

\section{Experimental preparation}

We used the giant neuron GCN (also known as MCC and C1) of Aplysia californica. Each cerebral ganglion contains a symmetrical pair of these cells. The axon bifurcates within a millimeter of the cell body (see Fig. 1 in Aletta and Goldberg, 1984). One branch is in the ipsilateral cerebrobuccal connective (CBC) and innervates the buccal ganglia and buc- 
Table 1. Size of the pool of serotonergic vesicles in the cell body of GCN after proximal transection of one branch of the bifurcate axon

\begin{tabular}{ll} 
Control & Experimental \\
\hline 99 & 128 \\
43 & 36 \\
83 & 82
\end{tabular}

The number of serotonergic vesicles per $100 \mu \mathrm{m}^{2}$ in the cell bodies of both GCNs in each of 3 ganglia was determined by electron microscopy as described in Materials and Methods. The CBC on one side of each ganglion was transected near the ganglion $3 \mathrm{~d}$ before fixation of the ganglion.

cal mass (Weiss et al., 1978). The other branch is in the ipsilateral posterior lip nerve (PLN) and presumably innervates lip musculature.

\section{Transection of nerves}

All transections were done in anesthetized animals through a small incision in the ventral surface of the head, which was then sutured (Aletta and Goldberg, 1984). Animals resumed normal activity within several hours of being returned to their holding tanks containing aerated artificial sea water. For proximal transections, the $\mathrm{CBC}$ and, in some cases, the PLN were severed about a millimeter from the cerebral ganglion. Distal transections or crushes were made about $10 \mathrm{~mm}$ from the ganglion. Transections were done on only one side of the ganglion, with the other GCN being used as a control. We would expect this other GCN to be the best control cell (as compared with GCNs from other animals), and, in fact, we have previously found that various parameters, such as rate of export of ${ }^{3} \mathrm{H}$-serotonin, vary much less between GCNs in a ganglion than between GCNs in different ganglia.

\section{Electron microscopy}

One $\mathrm{CBC}$ was transected proximally. Three days later the cerebral ganglion was removed, pinned in a dish containing supplemented artificial sea water, and then processed for electron microscopy. The pair of GCNs in 3 cerebral ganglia was analyzed. Two of the ganglia were processed using the technique of Cleary (1984), with the ganglia being fixed in $2 \%$ glutaraldehyde- $2 \%$ formaldehyde in $0.1 \mathrm{~m}$ cacodylate buffer and postfixed in $1 \%$ osmium tetroxide. The other ganglion was fixed in $6 \%$ glutaraldehyde in $0.2 \mathrm{~m}$ S-collidine and then postfixed in osmium tetroxide (Thompson et al., 1976). Tissue was dehydrated, embedded, and thin-sectioned. Sections were examined and photographed using a Phillips 300 electron microscope. Thin sections were taken from 3 regions of each GCN cell body: at the level of the middle of the nucleus and on both sides of the nucleus (Aletta and Goldberg, 1982). For each region, 24 randomly chosen $4.5 \times 2.7 \mu \mathrm{m}$ areas of cytoplasm were photographed, and prints were made at a final magnification of $52,000 \times$. Vesicles were counted in each micrograph by a person unaware of its provenance.

\section{Injection of ${ }^{3} \mathrm{H}$-fucose into $\mathrm{GCN}$}

The cerebral ganglion and its attached nerves were removed from the animal $1 \mathrm{~d}$ or $2 \mathrm{wk}$ after unilateral nerve transection or crush. Equal amounts of a concentrated solution of ${ }^{3} \mathrm{H}$-fucose $(67 \mathrm{Ci} / \mathrm{mmol}$; Amersham) were injected into the 2 GCN cell bodies by application of air pressure to calibrated double-barreled glass microelectrodes (Goldberg et al., 1976). The preparation was then kept at room temperature (21$23^{\circ} \mathrm{C}$ ) in artificial sea water supplemented with amino acids, vitamins, and glucose (Eisenstadt et al., 1973). After $6 \mathrm{hr}$, the CBCs and PLNs were detached from the ganglion and transferred to microhomogenizers containing electrophoresis buffer. ${ }^{3} \mathrm{H}$-glycoprotein is restricted to the axon of GCN in these nerves (Ambron et al., 1980). The cell bodies of the GCNs were harvested by microdissection in cold $70 \%$ ethylene glycol- $30 \%$ supplemented artificial sea water and also transferred to buffer.

\section{Gel electrophoresis}

Samples were electrophoresed on $7.5 \%$ polyacrylamide gel slabs according to the method of Laemmli (1970). The gels were then infiltrated with sodium salicylate (Chamberlain, 1979), dried, and exposed at $-80^{\circ} \mathrm{C}$ to Kodak X-Omat AR film that had been sensitized by exposure to a flash of light (Laskey and Mills, 1975). Fluorographs were scanned using

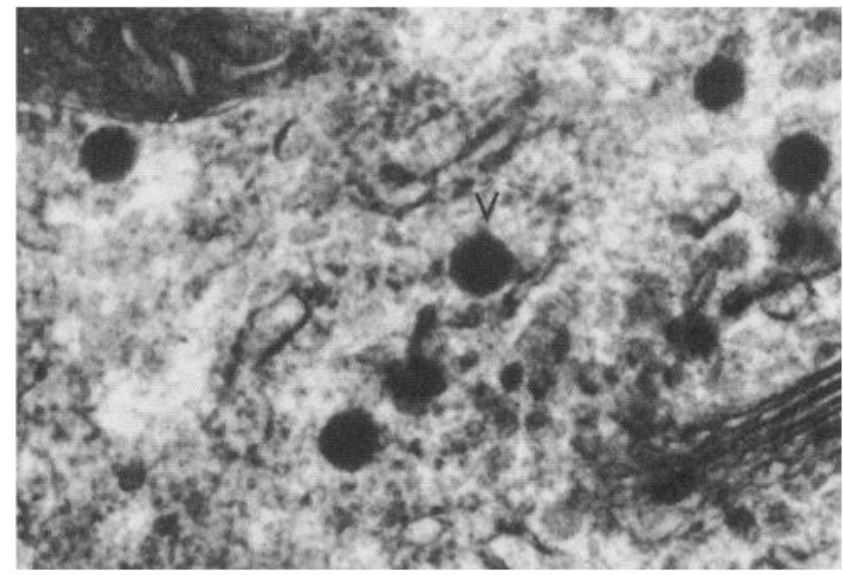

Figure 1. Serotonergic vesicles, darkly stained, in the cell body of $\mathrm{GCN}$. One of the 5 vesicles in this electron micrograph is labeled $(V)$. Magnification, $\times 52,000$.

a Joyce-Loebel microdensitometer. ${ }^{3} \mathrm{H}$-fucose in individual glycoproteins was quantitated by demarcating each peak on the densitometric scan by connecting its troughs with a line, cutting out the peak and weighing it. For the experiments in which both the CBC and PLN were transected proximally, the amounts of ${ }^{3} \mathrm{H}$-fucose in the $75 \mathrm{kDa}$ glycoprotein in the GCNs of a pair were normalized to adjust for differences in the total amounts of trichloroacetic acid-precipitable radioactivity in the cells.

\section{Fluorescence microscopy}

The CBC and, sometimes, the PLN were transected or crushed a few millimeters from the cerebral ganglion. After 4 or $12 \mathrm{~d}$, the ganglion was removed from the animal, and a $5 \%$ solution of Lucifer Yellow was pressure-injected into the cell body of GCN. The ganglion was kept at room temperature in supplemented artificial sea water for an hour. It was then fixed with $4 \%$ paraformaldehyde, dehydrated with ethanol, put in xylene, and then mounted with Entolin. The whole-mount was viewed with a Leitz Dialux 20EB fluorescence microscope using a blue excitation filter.

\section{Results}

\section{Number of serotonergic vesicles in cell body of $G C N 3$ after proximal transection of $C B C$}

Proximal transection of the $\mathrm{CBC}$, which contains one of GCNs 2 main axon branches, causes a proportionate decrease in the export of serotonergic storage vesicles from the cell body (number entering the axon per unit time), despite the fact that the intact branch of the axon can - and initially does - transport the vesicles that ordinarily would have traveled in the extirpated branch as well as its own normal complement of vesicles (Aletta and Goldberg, 1982). This precise reduction begins within hours and is completed within $3 \mathrm{~d}$.

The cell body of a normal GCN contains a large number of serotonergic vesicles (Aletta and Goldberg, 1982; Goldberg et al., 1978), which appear as dense-core vesicles in electron micrographs (Fig. 1). We were thus able to determine whether, when the reduction of export was completed, the size of the somal pool of vesicles had changed. We detected no substantial difference between the numbers of serotonergic vesicles in the cell bodies of the control and axotomized GCNs in each of 3 ganglia (Table 1).

\section{Incorporation of ${ }^{3} \mathrm{H}$-fucose into a putative vesicle glycoprotein after partial or complete axotomy}

The fact that the number of vesicles in the cell body of GCN did not increase at a time when the rate of their exit from the 


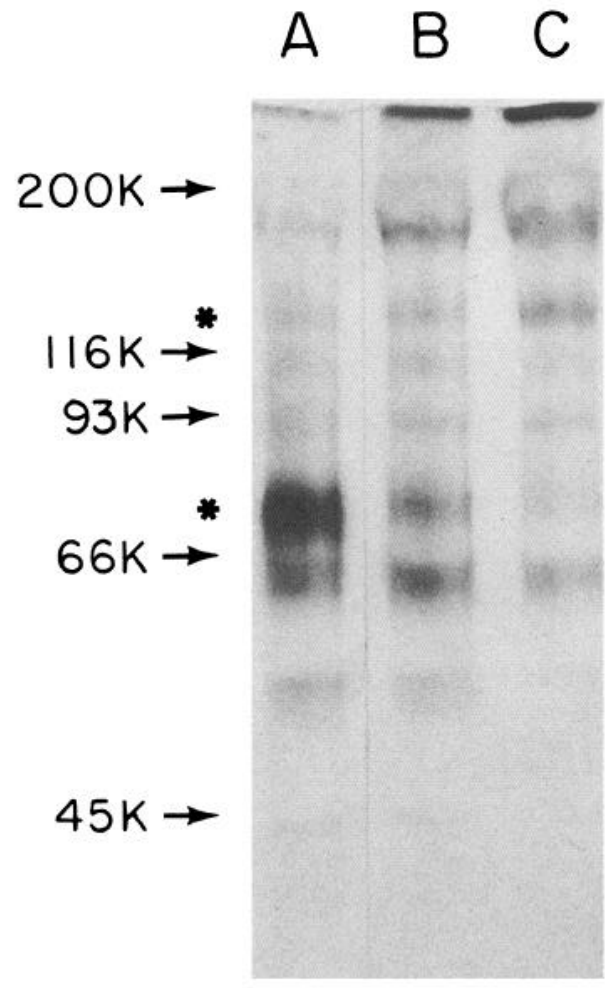

Figure 2. Fluorograph of ${ }^{3} \mathrm{H}$-fucosyl glycoproteins rapidly transported in the axon of GCN. Cells were labeled by microinjection of ${ }^{3} \mathrm{H}$-fucose into the cell body, followed $6 \mathrm{hr}$ later by homogenization of the nerves containing the axon branches of GCN. Proteins from the combined nerves were separated by one-dimensional SDS-PAGE (see Materials and Methods), with the entire samples being loaded onto the gel. ${ }^{3} \mathrm{H}$ fucosyl glycoproteins in the axons of normal GCNs are shown in lanes $A$ and $B$. The profile in lane $C$ is of an axon both of whose branches were crushed distally 2 wk prior to labeling. This GCN was in the same ganglion as the GCN of lane $B$, and equal amounts of ${ }^{3} \mathrm{H}$-fucose were injected into the 2 cells. (Lane $A$ should not be compared quantitatively with lanes $B$ and $C$, since it represents a GCN, from a different animal, that was injected with a different amount of ${ }^{3} \mathrm{H}$-fucose.) Arrows point to the positions of the designated molecular-weight markers, and asterisks denote the positions of the 130 and $75 \mathrm{kDa}$ glycoproteins.

cell body had been halved suggests that the rate of vesicle synthesis in the cell body decreases after axotomy. We thus sought to study the synthesis of a vesicle protein. Evidence indicates that a fucosyl glycoprotein whose apparent $M_{\mathrm{r}}$ during SDSPAGE is about $75,000 \mathrm{Da}$ is a constituent of the membrane of the serotonergic vesicle of GCN (Ambron et al., 1980, 1981; Cleary, 1984). Glycoproteins containing fucose are transported in the axon of GCN in serotonergic vesicles (Ambron et al., 1980; Cleary, 1984), and Figure 2 shows that the $75 \mathrm{kDa}$ glycoprotein and another of slightly smaller apparent $M_{\mathrm{r}}(\approx 65,000$ $\mathrm{Da})$ are the predominant ${ }^{3} \mathrm{H}$-fucosyl glycoproteins in the axon. (The bands are somewhat diffuse due to the presence of oligosaccharide moieties.) When both the CBC and PLN were transected far from the cerebral ganglion, the amount of the $75 \mathrm{kDa}$ ${ }^{3} \mathrm{H}$-glycoprotein appearing in the axon decreased (compare lane $\mathrm{C}$ in Fig. 2 with lane B, its paired control). In contrast, for example, the transport of the $130 \mathrm{kDa}{ }^{3} \mathrm{H}$-glycoprotein increased.

Was the diminished export of the $75 \mathrm{kDa}{ }^{3} \mathrm{H}$-glycoprotein caused by a decrease in its synthesis? We measured incorporation of injected ${ }^{3} \mathrm{H}$-fucose into proteins $24 \mathrm{hr}$ after proximal transection of the $\mathrm{CBC}$ only. A $6 \mathrm{hr}$ period of incorporation was selected as the minimal period for sufficient label to be consistently incorporated. Figure 3, representative of 4 experiments,

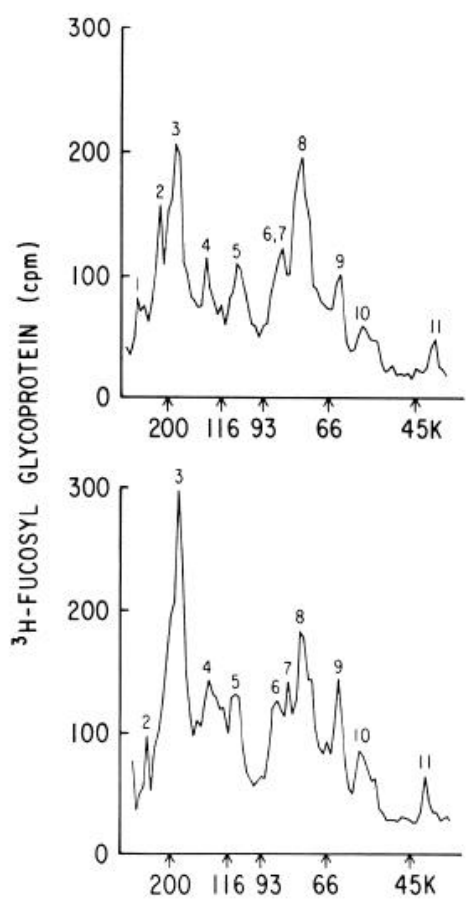

Figure 3. Gel profiles of ${ }^{3} \mathrm{H}$-fucosyl glycoproteins in the combined cell body and axon of a normal GCN (top) and of the other GCN in the ganglion, whose $\mathrm{CBC}$ axon branch was proximally transected $24 \mathrm{hr}$ prior to labeling (bottom). Labeling and electrophoresis were performed as described in the legend to Figure 2. Equal amounts of ${ }^{3} \mathrm{H}$-fucose were injected into the 2 cells. The gels were sectioned sequentially into $1 \mathrm{~mm}$ pieces for counting of radioactivity by liquid scintillation.

shows that axotomy resulted in a decrease in the amount of ${ }^{3} \mathrm{H}$ fucose in the $75 \mathrm{kDa}$ glycoprotein (no. 8) relative to the amount in the other glycoproteins. (In this experiment, there was a 33\% decrease in the amount of ${ }^{3} \mathrm{H}$-fucose in glycoprotein no. 8 after normalization for total incorporated ${ }^{3} \mathrm{H}$-fucose in the lanes.) As expected, proximal transection of both the PLN and the CBC exaggerated the effect. In each of 4 experiments a profound, selective decrease in the $75 \mathrm{kDa}$ glycoprotein was observed (Fig. 4). The ratio of ${ }^{3} \mathrm{H}$-fucose in the $75 \mathrm{kDa}$ glycoprotein in the control cell to ${ }^{3} \mathrm{H}$-fucose in that protein in the paired axotomized cell was $4.3 \pm 1.3$ (mean \pm SEM). A considerable decrease in the content of ${ }^{3} \mathrm{H}$-fucose was also seen in a protein of slightly larger apparent $M_{\mathrm{r}}(82,000 \mathrm{Da})$. This protein was never observed in the axon, however (Figs. 2, 6).

\section{Partitioning of transported ${ }^{3} \mathrm{H}$-fucosyl glycoproteins} between the 2 axon branches

In a normal GCN, about $70 \%$ more ${ }^{3} \mathrm{H}$-fucosyl glycoprotein and ${ }^{3} \mathrm{H}$-serotonin is transported in the CBC than in the PLN (Aletta and Goldberg, 1982, 1984), and none of the individual major glycoproteins partitions consistently differently from the others; that is, none shows a consistent preference for the PLN (Ambron et al., 1980). Within hours after transection of the CBC relatively far from the cerebral ganglion, the majority of the serotonergic vesicles that would ordinarily be transported by that branch instead enter the PLN branch, despite the continued ability of the transected branch to transport its normal complement of vesicles (Aletta and Goldberg, 1984). Thus, the partitioning of vesicles is strongly influenced in this situation by the states of the endings of the branches. Figure 5, a fluorescence micrograph of the CBC and PLN filled with Lucifer yellow, shows that there is abundant growth from both after they are transected. Similar growth from the CBC branch also occurs if it alone is cut or if the nerves are crushed (data not shown). We could thus measure 
partitioning of transported material between a branch with growing sprouts (CBC branch) and a branch of the same axon with synaptic endings (PLN branch), although we could not exclude the possibility that growth was also occurring from the PLN branch even though it was uninjured.

We wanted to see whether other transported fucosyl membrane glycoproteins partitioned like the $75 \mathrm{kDa}$ vesicle glycoprotein after transection of the $\mathrm{CBC}$ or, rather, if there was selectivity in partitioning. We studied the partitioning of the glycoproteins 2 wk after the $\mathrm{CBC}$ had been transected distally. At this time, $70 \%$ of the serotonin, rather than the normal $36 \%$, is transported in the PLN (Aletta and Goldberg, 1984). Were there selectivity in the partitioning of transported material, we would expect to have observed consistent differences in the gel profiles of the CBC and PLN, that is, either a consistent enrichment of the $75 \mathrm{kDa}$ glycoprotein relative to the other glycoproteins in the PLN or a consistent enrichment of another of the glycoproteins in the CBC. Gel profiles from 5 experiments showed that there was no major difference in the glycoprotein composition of the CBC and PLN. In each pair there were some differences, however. In the pair shown in Figure $6 \mathrm{~A}$, for example, the $130 \mathrm{kDa}$ glycoprotein (no. 4) partitioned more equally between the 2 branches than did the $75 \mathrm{kDa}$ glycoprotein (no. $8)$. However, neither this difference nor any other was observed consistently (Fig. 6B). Had all of the glycoproteins partitioned in the same proportions, all of the glycoprotein symbols would approximately superimpose in each of the 5 experiments represented in Figure $6 B$. Instead, there was considerable spread in each experiment. As is clear from the frequency of line intersections in the graph, however, the differences in partitioning were not consistent from one axon to the next. For example, in some experiments glycoprotein no. 4 was underrepresented in the PLN (experiments 1-3), while in others it was overrepresented (experiments 4 and 5). The same can be said of the 75 $\mathrm{kDa}$ glycoprotein (no. 8) and the others.

\section{Discussion}

\section{Decreased export of transmitter vesicles after axotomy}

The results reported here and previously (Aletta and Goldberg, 1982,1984 ) indicate that axotomy of a neuron should be a useful tool for addressing questions of organelle biogenesis not readily approached with other types of cells. Because of the unusual geometry of the neuron, the sites of use of the transmitter storage vesicle usually are distant from the site of synthesis. Thus, some or most of the sites of use can be removed without directly affecting the site of synthesis. It was found previously that such removal results in a precisely graded decrease in the export of vesicles by the cell body (Aletta and Goldberg, 1982). Therefore, we have a convenient method for studying how a cell modulates its output of a secretory organelle.

The decrease in the rate of export after partial or complete axotomy must be due either to a decrease in the net rate of vesicle production, or to a decrease in the rate at which vesicles are readied for export. The data reported here support the previous suggestion that production is likelier to be the regulated step (Aletta and Goldberg, 1984). Were the rate of export to decrease without a commensurate decrease in the rate of production, vesicles would accumulate in the cell body. This increase in number should be substantial, because we estimate that about one-quarter of the somal pool of vesicles is exported per hour (Aletta and Goldberg, 1982, 1984). Yet, when we counted the number of serotonergic vesicles in the cell body, we did not observe a substantial difference between axotomized and control cells.

In addition, we found that the incorporation of ${ }^{3} \mathrm{H}$-fucose into a $75 \mathrm{kDa}$ putative vesicle protein, but not into other transported glycoproteins, decreased after axotomy. This protein is thought

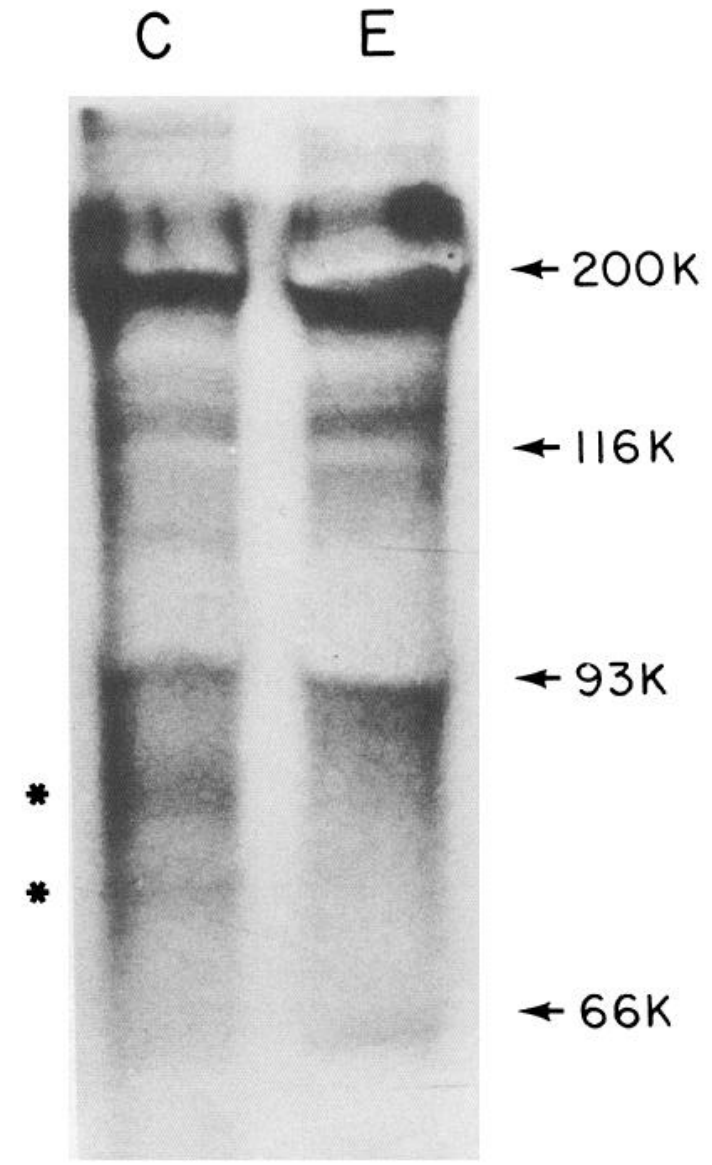

Figure 4. Fluorograph of ${ }^{3} \mathrm{H}$-fucosyl glycoproteins in the combined cell body and axon of a normal GCN $(C)$ and of the other GCN in the ganglion, whose CBC and PLN axon branches were proximally transected $24 \mathrm{hr}$ prior to labeling $(E)$. Labeling and electrophoresis were as described in the legend to Figure 2. Asterisks denote the positions of the 82 and $75 \mathrm{kDa}$ glycoproteins.

to be a constituent of the serotonergic vesicle because ${ }^{3} \mathrm{H}$-fucosyl glycoproteins move by fast transport in the axon of GCN predominantly in the vesicle (Cleary, 1984) and this is the predominant fucosyl glycoprotein in the axon (Fig. 2; see also Ambron et al., 1980), and because this protein copurifies with vesicles during subcellular fractionation (Ambron et al., 1981). By measuring incorporation of ${ }^{3} \mathrm{H}$-fucose, we were presumably observing a step close to the actual formation of the vesicle, since vesicles bud from the Golgi apparatus (Ambron et al., 1980; Cleary, 1984; Hammerschlag et al., 1982; Jamieson and Palade, 1977; Thompson et al., 1976) and fucosylation is thought to occur late in Golgi processing (Leblond and Bennett, 1977).

Our data are compatible with decreased export after axotomy being caused by increased degradation of vesicles in the cell body rather than decreased synthesis, though this would seem to be a wasteful mechanism of regulation. We also think it unlikely because we observed large decreases in the amount of ${ }^{3} \mathrm{H}$-fucose in the putative vesicle glycoprotein during a relatively brief labeling period. It would be difficult to measure directly the rate of disappearance of ${ }^{3} \mathrm{H}$-fucose from this protein because so little is detectable in the protein when the effect is maximized by transecting both axon branches.

The lack of an increase in the somal pool of vesicles dovetails with the decrease in fucosylation of the putative vesicle protein in supporting the idea that synthesis, rather than mobilization for export, is the regulated step. Consideration of the origin of the somal pool in a normal GCN, however, makes it puzzling 


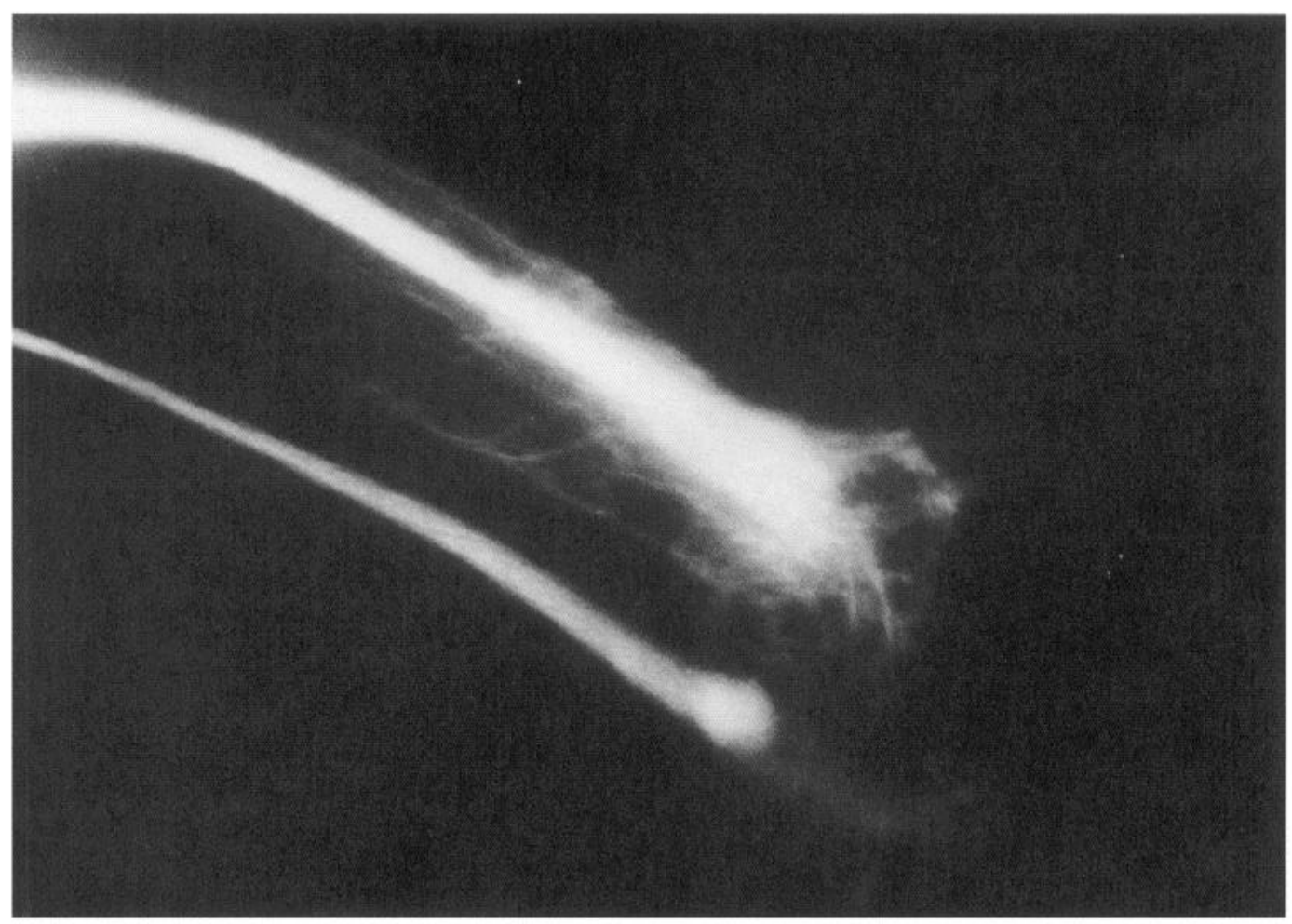

Figure 5. Sprouting from the end of the transected axon of GCN. Sprouts from the CBC branch are easily visible; those from the PLN branch, which was also transected, are mainly out of focus. Lucifer Yellow was injected into the cell body of GCN $12 \mathrm{~d}$ after transection. Magnification, $\times 250$.

that the somal pool did not diminish after axotomy. There might normally be a somal pool because the number of vesicles ready to exit the cell body might exceed the capacity of the transport machinery. Or vesicles might only randomly come into contact with the machinery, with the probability of contact sufficiently low such that it takes appreciable time for a typical vesicle to exit. Or each vesicle might have to go through an appreciably time-consuming assembly line of processing steps before being competent to exit. All three possibilities, when interpreted as simply as possible, predict a decrease in the size of the somal pool when the rate of formation of vesicles decreases. One explanation for the lack of decrease in pool size that occurs to us is that newly synthesized vesicles are preferentially and rapidly exported from the cell body, but not all of them manage to exit. Those that do not quickly exit join a large somal pool whose members exit much more slowly (Goodrum and Morell, 1984). Were this true, a decrease in the rate of vesicle formation would cause a decrease in the rate of export without substantially diminishing the somal pool, at least until well after the regulatory event.

Because axotomy appears to be a way of eliciting a regulated reduction in the formation of a secretory organelle, and little is known about how a cell achieves changes in rates of synthesis of organelles, it would be of considerable interest to know whether the synthesis of other vesicle proteins is reduced by axotomy. Need there be coordinate regulation of the synthesis of many vesicle proteins or of those unique to the vesicle? If there were, axotomy would provide a technique for rapidly identifying proteins with a reasonable likelihood of residing in the vesicle.

\section{Partitioning of fucosyl glycoproteins between}

2 branches of the axon after distal transection of 1 branch

While different amounts of material may enter individual branches of an axon by fast axonal transport (Brimijoin et al.,
1980; Lasek, 1968; Ochs, 1972) because of differences in the caliber of the branches or the state of the branch endings (Aletta and Goldberg, 1984), there is no clear evidence that the composition of the material differs. Several studies failed to show selectivity in the partitioning of transported material (Bisby, 1981; Perry and Wilson, 1981; Stone and Wilson, 1979). A single cell with bifurcate axon, like GCN, is the ideal preparation in which to look for selectivity, because the partitioning differences that have been observed in multineuron preparations (Rulli and Wilson, 1985) may reflect not intracellular selectivity, but intercellular differences in anatomy, biochemistry, or physiology.

We found no evidence of consistent selectivity in the partitioning of the 6 newly synthesized fucosyl glycoproteins that we studied in detail. Even glycoprotein no. 4 (Fig. 6), whose transport seemed to increase moderately after axon transection, and which thus might play a role in the recovery of the axon from injury, was not preferentially shunted into the injured branch. Our data, which constitute the first such study using a single neuron, agree with those previously reported by Bisby (1981) and Perry and Wilson (1981), who used mammalian multineuron preparations. A conclusion that there is no selectivity in partitioning is tentative now, however, because we studied only a small group of proteins.

Our results indicate, though, that during a relatively short time the composition of the mixture of newly synthesized glycoproteins received by the ends of a branch of an axon can differ substantially from the composition of the mixture received by the ends of another branch. (Some of these differences might be illusory, because we cannot be certain that the specific activities of an individual glycoprotein were consistently similar in the two branches.) This might be due to random variation in the partitioning of organelles at a branch point; that is, one would expect fluctuations in the partitioning ratios for individual organelle types from one relatively brief period to the next, much 


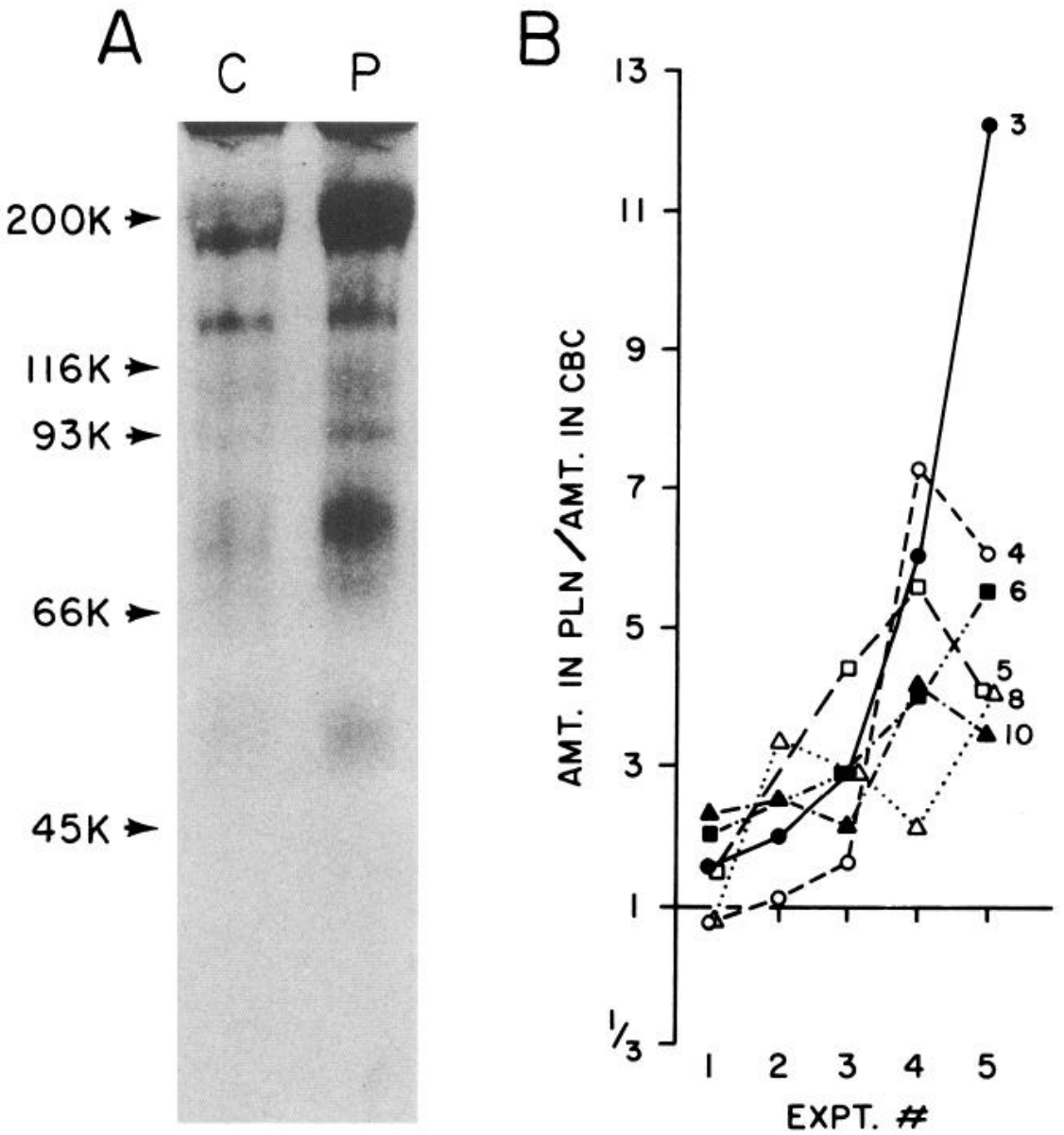

Figure 6. Partitioning of rapidly transported ${ }^{3} \mathrm{H}$-fucosyl glycoproteins between the CBC and PLN axon branches of GCN 2 wk after transection of the CBC far from the bifurcation. Labeling and electrophoresis were as described in the legend to Figure 2. $A$, Fluorograph of the ${ }^{3} \mathrm{H}-\mathrm{fu}$ cosyl glycoproteins in the $\mathrm{CBC}$ branch $(C)$ and the PLN branch $(P)$ of the GCN of experiment 3. B, Partitioning ratios, determined from densitometric scanning of the autoradiograms, for 6 fucosyl glycoproteins in 5 separate experiments. The relative areas under the peaks were determined by cutting and weighing. as 10 coin tosses might once yield 3 heads, once 6 , and once 4 . Variability in the composition of an individual type of organelle could also contribute; for example, serotonergic storage vesicles might differ in the molecular ratios of their normal component proteins, not by design but rather as an expression of tolerance in their manufacture. Whether variability in the receipt of material by the branches is of functional significance is a question that merits further study.

\section{References}

Aletta, J. M., and D. J. Goldberg (1982) Rapid and precise down regulation of fast axonal transport of transmitter in an identified neuron. Science 218: 913-916.

Aletta, J. M., and D. J. Goldberg (1984) Routing of transmitter and other changes in fast axonal transport after transection of one branch of the bifurcate axon of an identified neuron. J. Neurosci. 4: 18001808.

Ambron, R. T., J. E. Goldman, L. J. Shkolnik, and J. H. Schwartz (1980) Synthesis and axonal transport of membrane glycoproteins in an identified serotonergic neuron of Aplysia. J. Neurophysiol. 43: 929-944.

Ambron, R. T., A. A. Sherbany, L. J. Shkolnik, and J. H. Schwartz (1981) Distribution of membrane glycoproteins among the organelles of a single identified neuron of Aplysia. I. Association of a $\left[{ }^{3} \mathrm{H}\right]$ glycoprotein with vesicles. Brain Res. 207: 17-32.

Bisby, M. A. (1981) Axonal transport in the central axon of sensory neurons during regeneration of their peripheral axon. Neurosci. Lett. 21: 7-11.

Boyle, C., and J. S. Gillespie (1970) Accumulation and loss of noradrenaline central to a constriction of adrenergic nerves. Eur. J. Pharmacol. 12: 77-84.

Brimijoin, S., J. M. Lundberg, E. Brodin, T. Hökfelt, and G. Nilsson (1980) Axonal transport of substance $P$ in the vagus and sciatic nerves of the guinea pig. Brain Res. 191: 443-457.
Chamberlain, J. P. (1979) Fluorographic detection of radioactivity in polyacrylamide gels with the water-soluble fluor, sodium salicylate. Anal. Biochem. 98: 132-135.

Cleary, L. J., Jr. (1984) Identification of the axonal organelles in which newly-synthesized glycoproteins are translocated by fast axonal transport in the giant cerebral neuron of Aplysia. Ph.D. thesis, Columbia University, New York.

Eisenstadt, M., J. E. Goldman, E. R. Kandel, H. Koike, J. Koester, and J. H. Schwartz (1973) Intrasomatic injection of radioactive precursors for studying transmitter synthesis in identified neurons of Aplysia californica. Proc. Natl. Acad. Sci. USA 70: 3371-3375.

Giulian, D., H. Des Ruisseaux, and D. Cowburn (1980) Biosynthesis and intra-axonal transport of protein during neuronal regeneration. J. Biol. Chem. 255: 6494-6501.

Goldberg, D. J., and R. T. Ambron (1985) Consequences of partial axotomy for production of neurotransmitter vesicles and routing of material moving by fast transport in the axonal tree. Soc. Neurosci. Abstr. 11: 420.

Goldberg, D. J., J. E. Goldman, and J. H. Schwartz (1976) Alterations in amounts and rates of serotonin transported in an axon of the giant cerebral neurone of Aplysia californica. J. Physiol. (Lond.) 259: 473490.

Goldberg, D. J., J. H. Schwartz, and A. A. Sherbany (1978) Kinetic properties of normal and perturbed axonal transport of serotonin in a single identified axon. J. Physiol. (Lond.) 281: 559-579.

Goodrum, J. F., and P. Morell (1984) Explanation of the apparent biphasic axonal transport kinetics of fucosylated glycoproteins. J. Neurosci. 4: 1830-1839.

Hammerschlag, R., G. C. Stone, F. A. Bolen, J. D. Lindsey, and M. H. Ellisman (1982) Evidence that all newly synthesized proteins destined for fast axonal transport pass through the Golgi apparatus. J. Cell Biol. 93: 568-575.

Hebb, C. O., and A. Silver (1966) Axoplasmic flow of protein. In Protides of the Biological Fluids, Vol. 13, H. Peeters, ed., pp. 179180, Elsevier, Amsterdam. 
Jamieson, J. D., and G. E. Palade (1977) Production of secretory proteins in animal cells. In International Cell Biology, B. R. Brinkley and K. R. Porter, eds., pp. 308-317, Rockefeller U. P., New York.

Laemmli, V. K. (1970) Cleavage of structural proteins during the assembly of the head of bacteriophage T4. Nature [New Biol.] 227: 680-685.

Lasek, R. J. (1968) Axoplasmic transport in cat dorsal root ganglion cells: As studied with ${ }^{3} \mathrm{H}-\mathrm{L}-\mathrm{leucine}$. Brain Res. 7: 360-377.

Laskey, R. A., and A. D. Mills (1975) Quantitative film detection of ${ }^{3} \mathrm{H}$ and ${ }^{14} \mathrm{C}$ in polyacrylamide gels by fluorography. Eur. J. Biochem. 56: 335-341.

Leblond, C. P., and G. Bennett (1977) Role of the Golgi apparatus in terminal glycosylation. In International Cell Biology, B. R. Brinkley and K. R. Porter, eds., pp. 326-336, Rockefeller U. P., New York.

Ochs, S. (1972) Rate of fast axoplasmic transport in mammalian nerve fibres. J. Physiol. (Lond.) 227: 627-645.

Perry, G. W., and D. L. Wilson (1981) Protein synthesis and axonal transport during nerve regeneration. J. Neurochem. 37: 1203-1217.
Rulli, R. D., and D. L. Wilson (1985) Proteins in fast axonal transport are differentially transported in branches of sensory nerves. Brain Res. 335: $165-168$.

Skene, J. H. P., and M. Willard (1981) Changes in axonally transported proteins during axon regeneration in toad retinal ganglion cells. $J$. Cell Biol. 89: 86-95.

Stone, G. C., and D. L. Wilson (1979) Quantitative analysis of proteins rapidly transported in ventral horn motoneurons and bidirectionally from dorsal root ganglion. J. Neurobiol. 10:1-12.

Thompson, E. B., J. H. Schwartz, and E. R. Kandel (1976) A radioautographic analysis in the light and electron microscope of identified Aplysia neurons and their processes after intrasomatic injection of $\mathrm{L}-\left[{ }^{3} \mathrm{H}\right]$ fucose. Brain Res. $112: 251-281$.

Weiss, K. R., J. L. Cohen, and I. Kupfermann (1978) Modulatory control of buccal musculature by a serotonergic neuron (metacerebral cell) in Aplysia. J. Neurophysiol. 41: 181-203. 